\title{
The Effect of the Immunosuppressive Agent on Scleroderma in a Graft Versus Host Disease (GVHD) Model Using an X-ray Irradiation Device in Mice
}

\author{
Shiruku Hosoi, Shinichi Ozato, Tatsumi Wada, Takumi Yamazawa, Shinichiro Tsunesumi, \\ Seiichi Katayama, Katsuhide Nishi, Naoyuki Hironaka
}

Pharmacology Department, Kumamoto Laboratory, LSI Medience Corporation, Japan

The graft versus host disease (GVHD) is known as the complication which is the transplanted donner cells attacks patient's organ immunologically after the bone marrow transplantation. Because of serious problems in the clinical scene, it is required to develop effective drugs to treat these symptoms. Therefore, we tried to develop the animal model of the GVHD to evaluate effectiveness of novel agents for clinical use. It is known that the GVHD-like symptoms occurs by applying an X-ray and transplanting bone marrow cells of other strain's mice to a mouse. In the previous study, we introduced a device that is simple and capable of irradiating of the X-ray in mice to establish the model of the allogenic GVHD. We used the method in the previous study to ensure the reproducibility of the model. In this study, in order to confirm the usefulness of the present mode, we administered the immunosuppressive agents to confirm the usefulness of the model. After 28 days from the X-ray irradiation and transplantation of the other strain mice cells, the remarkable skin thickening was observed on a back skin of the mice, which suggests that the model develops a scleroderma. The prednisolone, one of the immunosuppressive agents which in use of clinical GVHD treatment, suppressed the thickening of the skin in the model. The result in this study implies that the X-ray irradiation induced GVHD model in mice is a reproducible and it could be used to evaluate the effect of therapeutic agents. 